\title{
WARTen AUf Gestern: \\ ZUKUNFTSPERSPEKTIVEN SYRISCHER FLÜCHTLINGE UND DAUERHAFTE LÖSUNGEN DER UNO
}

Ronald STADE, Faculty of Culture and Society, Universität Malmö, Schweden Lana STADE, Durable Solutions Platform (DSP), Amman/Jordanien

\section{INHALT}

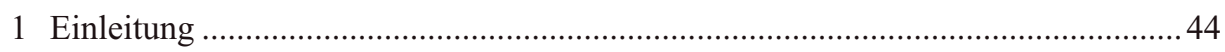

2 Die Lage in Syriens Nachbarstaaten ..................................................................... 46

2.1 Zahlenmäßige Dimensionen der Fluchtmigration .................................................46

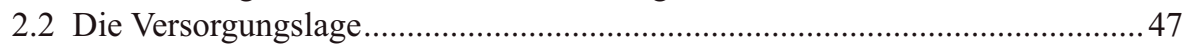

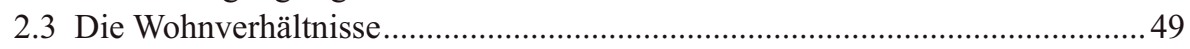

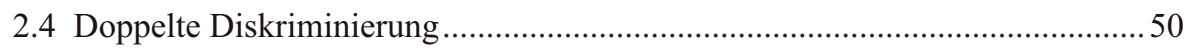

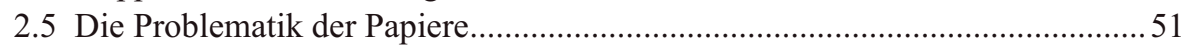

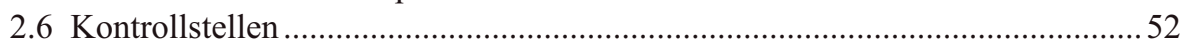

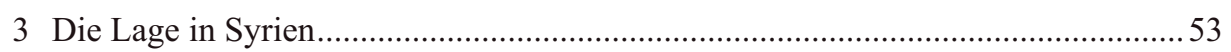

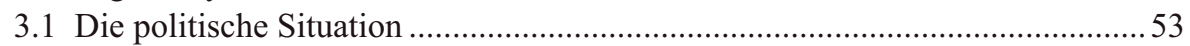

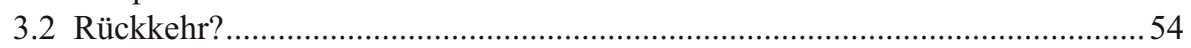

3.3 Umfragen zu den Absichten syrischer Flüchtlinge............................................54

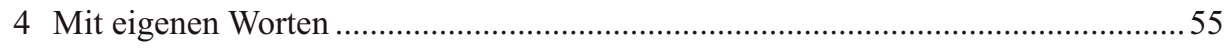

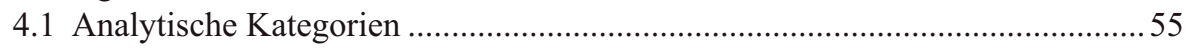

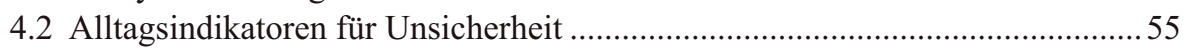

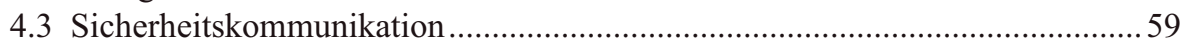

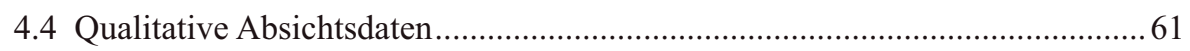

5 Alltagsindikatoren, Kommunikation, Absichten und dauerhafte Lösungen ..............63

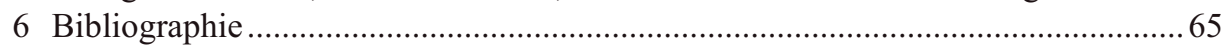

„Wir würden zurückgehen nach Syrien, wenn alles so wäre wie vor dem Krieg. Wir wollen, dass alles so ist wie vorher. Wir hatten ein so gutes Leben vorher." 


\section{Einleitung}

Obiges Zitat stammt aus den Gesprächen, die wir im Rahmen unseres Forschungsprojekts ,, What Future for Syrian Refugees? “ mit syrischen Flüchtlingen geführt haben. ${ }^{1}$ In diesem Projekt ging es darum zu verstehen, wie syrische Flüchtlinge in den Nachbarländern Syriens ihre eigene Situation einschätzen, welche Zukunftsperspektiven sie entwickeln - und auf Grundlage welcher Kommunikation und Information sie das tun und inwieweit eine Rückkehr nach Syrien Teil dieser Perspektiven ist. Mit empirischen Methoden wollten wir dazu drei miteinander zusammenhängende Fragen mit Hilfe von übergeordneten analytischen Begriffen beantworten:

1. Alltagsindikatoren für Unsicherheit: Wie definieren syrische Flüchtlinge in Libanon, Jordanien und der Türkei Gefährdungen und Unsicherheit sowohl an ihrem jetzigen Wohnort als auch in Syrien?

2. Sicherheitskommunikation: Welche Kommunikationskanäle und Informationen benutzen syrische Flüchtlinge in Libanon, Jordanien und der Türkei, um zu entscheiden ob die Situation innerhalb Syriens sicher und stabil ist?

3. Qualitative Absichtsdaten: Welche Hoffnungen, Absichten und Pläne haben syrische Flüchtlinge in Libanon, Jordanien und der Türkei, insbesondere in Bezug auf eine mögliche Rückkehr nach Syrien?

Wir verfolgen mit diesem Ansatz ein prinzipielles anthropologisches Anliegen. Statt einer herkömmlichen, oft impliziten Aufteilung zwischen den emischen Perspektiven des Insiders (d. i. des Beforschten) und den etischen Perspektiven des Outsiders (sprich des Forschers) möchten wir diesen Unterschied aufheben. Das möchten wir allerdings nicht dahingehend tun, dass wir, wie unter Anthropologen eher üblich, behaupten, dass alle Perspektiven, also auch solche, die vermeintlich etisch sind, letztendlich auch nur kulturspezifische emische Sichtweisen - nämlich die der Forscher - repräsentieren. Eine solche vulgäre Form des Relativismus endet oftmals in der unsinnigen Behauptung, dass es keine außerkulturelle Wirklichkeit und somit keine die eigenen kulturellen Annahmen und Normen überschreitende Perspektive gibt. Wir gehen davon aus, dass Menschen die Fähigkeit besitzen sich Gedanken über ihre Situation zu machen und dass sie sich dabei auch mitunter auf entfremdende Weise von außen betrachten. So verstehen z. B. syrische Flüchtlinge sehr wohl, dass sie oft nicht nur in ihren Aufnah-

Diese Studie wurde auf großzügige und unbürokratische Weise von dem schwedischen Riksbankens Jubileumsfond finanziert. Wesentliche Unterstützung bekamen wir auch von den zivilgesellschaftlichen Organisationen „Abaad“, „Himaya“ und „Nabad“, sowie von Frau Pascale Jalbout. Ohne die begabten und tüchtigen Moderatorinnen Lina Ashkar und Zeina Shoueib hätten wir unser Projekt nicht durchführen können. Schließlich sind wir den vielen Teilnehmern der Fokusgruppen Dank schuldig. Ihre Gastfreundschaft und Offenheit haben uns dazu verpflichtet, ihre Erfahrungen und Hoffnungen nach bestem Vermögen bekanntzumachen. 
meländern, sondern auch von der Weltgemeinschaft als Belastung aufgefasst werden. Sie haben auch Einsicht in die Absurdität ihrer globalen Position, dass sie nämlich in Armut leben müssen, während Menschen in anderen Teilen der Welt ein behagliches Dasein führen. Durch Kommunikation tragen sie Informationen über ihre jetzige Situation, die Lage in Syrien, die Rolle der am Krieg beteiligten Mächte und vieles anderes zusammen und analysieren diese. Man könnte also behaupten, dass nicht nur Forscher, sondern auch Beforschte sowohl emische als auch etische Perspektiven anlegen. Wir gestehen unseren syrischen Gesprächspartnern die generische Fähigkeit zu, ihr Leben auch von außen, sozusagen von nirgendwo, zu betrachten. ${ }^{2}$ Die emischen und etischen Perspektiven der Flüchtlinge bilden demzufolge nicht den Gegensatz zu theoretischen und politischen Analysen ihrer Situation, sondern beziehen diese mit ein.

Im abschließenden Teil dieses Beitrags werden wir die Perspektiven der syrischen Flüchtlinge mit den von der UNO vorgeschlagenen dauerhaften Lösungen von Vertreibung und Flucht vergleichen. Letztere lauten:

A. Freiwillige Rückkehr in Sicherheit und Würde,

B. Resettlement in ein Drittland oder

C. Integration im Aufnahmeland.

Namentlich sollen die Alltagsindikatoren für Unsicherheit, so wie sie von den syrischen Flüchtlingen selber beurteilt werden, mit den dauerhaften Lösungen der UNO verglichen werden um herauszufinden welche Herausforderungen es noch zu bewältigen gilt. Infolge der Sammlung empirischer Daten im Rahmen von Diskussionen mit syrischen Flüchtlingen in Fokusgruppen und Interviews mit Schlüsselinformanten konnten wir auf eine umfassende Datenmenge zurückgreifen, die wir während einer Basisstudie im Auftrag des Kinderhilfswerks der Vereinten Nationen (UNICEF) zusammengetragen hatten (MALMÖ UnIVERsity \& UNICEF 2017). Für diese Studie hatten wir 7.000 stundenlange Interviews mit Haushaltsmitgliedern, sowie 48 Fokusgruppen und 42 Interviews mit Schlüsselinformanten in allen Gouvernements Libanons durchgeführt. Davon entfielen 1.492 Haushaltinterviews und 20 Fokusgruppendiskussionen auf syrische Flüchtlinge. ${ }^{3}$

Siehe NAgel 1986 und RAPPORT \& STAde 2014 zur Frage des menschlichen Vermögens sich selbst aus entfremdender Perspektive zu betrachten.

3 Die UNICEF-Studie basierte auf einer Wahrscheinlichkeitsstichprobe. Der Stichprobenumfang (n) bei der Befragung syrischer Flüchtlinge betrug insgesamt 1.221.051. Der Probenahmefehler jeder Wahrscheinlichkeitsstichprobe wird folgendermaßen berechnet:

$\mathrm{E}=1.96 * \sqrt{(\mathrm{P} *}(1-\mathrm{P}) / \mathrm{n}) * \sqrt{(\text { deff })}$, wobei P der Prozentsatz des Interessenparameters, (n) der Stichprobenumfang und (deff) der Designeffekt durch Klumpung ist. 
Für die vorliegende Studie haben wir diese Erhebung durch 26 Fokusgruppen mit syrischen Flüchtlingen und 20 Interviews mit Schlüsselinformanten in Libanon vervollständigt. Basierend auf unseren vorherigen Erfahrungen trafen wir dabei eine bewusste Auswahl der Orte, an denen wir Fokusgruppen durchführten. Wir beachteten, in welchen Gebieten Libanons es eher leicht ist, syrische Flüchtlinge zu erreichen und mieden diese, da zu befürchten war, dass syrische Flüchtlinge in diesen Gegenden zur Genüge studiert worden waren und möglicherweise bereits an „Forschungsmüdigkeit“ litten. Stattdessen organisierten wir Fokusgruppen in unzugänglichen bzw. bislang wenig aufgesuchten Regionen, in denen eine erhebliche Zahl syrischer Flüchtlinge lebt. ${ }^{4}$ Für die Diskussionen in den Fokusgruppen benutzten wir einen Fragebogen mit Stichpunkten um komparative Daten zu erhalten. Jedoch leiteten wir die Moderatoren an, Unterbrechungen des Diskussionsflusses nach Möglichkeit zu vermeiden. Auf diese Art gewannen wir ein breites Spektrum an unvorhergesehenen Informationen, z. B. über die vielfältigen Fluchterfahrungen der Teilnehmer.

Im Folgenden beginnen wir mit einer Beschreibung der Ausgangslage für unsere Studie. Danach lassen wir die syrischen Flüchtlinge zu Wort kommen. Abschließend vergleichen wir die seitens der Flüchtlinge identifizierten Alltagsindikatoren für Unsicherheit mit den von der UNO vorgeschlagenen dauerhaften Lösungen für Vertriebene und Flüchtlinge. Letztendlich stellen wir fest, dass der tiefgreifende und folgenschwere Vertrauensmangel und die Auflösung des sozialen Zusammenhalts in Syrien, die auf den Verbleib des Regimes unter Baschar Assad zurückzuführen sind, wahrscheinlich die größte Herausforderung für eine dauerhafte Lösung darstellen.

\section{Die Lage in Syriens Nachbarstaaten}

\subsection{Zahlenmäßige Dimensionen der Fluchtmigration}

2011 schlug die Regierung Syriens Demonstrationen und Aufstände mit unangemessener Gewalt nieder, was dazu führte, dass die Aufständischen anfingen bewaffneten Widerstand zu leisten. Der Bürger- bzw. Stellvertreterkrieg, der daraus entstand, wird zum Zeitpunkt der Kompilation dieses Beitrags immer noch fortgeführt. Mindestens die Hälfte der Bevölkerung Syriens ist durch den Krieg vertrieben worden und über sechs Millionen Syrer sind über die Grenzen ins Ausland geflohen. Die Menschen in den Nachbarländern Syriens waren es schon seit den 1960er Jahren gewohnt hunderttausende Syrer als Arbeitsmigranten zu empfangen. Dies waren jedoch fast ausschließlich syrische Männer, die als Saisonarbeiter nach Libanon, Jordanien und in die Türkei kamen, um dann wieder nach Hause zu fahren. Nun kamen innerhalb kurzer Zeit Milli-

\footnotetext{
${ }^{4}$ Genauer gesagt haben wir neun Fokusgruppen in Akkar, acht im Bekaa, fünf in Mount Lebanon, zwei in Baalbek und zwei in Südbeirut durchgeführt.
} 
onen von syrischen Männern, Frauen und Kindern, mitunter ganze Dorfgemeinschaften, die viele Jahre später immer noch nicht in ihr Heimatland zurückgekehrt sind. Indem sie zirka 3,5 Millionen syrische Flüchtlinge aufgenommen hat, ist die Türkei das Land mit der weltweit größten Flüchtlingsbevölkerung. Im Verhältnis zur eigenen Bevölkerung ist jedoch Libanon das Land, welches die weltweit größte Zahl an Flüchtlingen beherbergt. Vor dem Ausbruch des Syrienkriegs hatte Libanon eine geschätzte Bevölkerung von 4,2 Millionen; seitdem sind fast zwei Millionen Flüchtlinge aus Syrien hinzugekommen. ${ }^{5}$ Niemand weiß genau wie viele syrische Flüchtlinge sich in Jordanien aufhalten. Das Flüchtlingskommissariat der UNO (UNHCR) hat 673.414 Flüchtlinge registriert, ${ }^{6}$ aber die jordanische Regierung schätzt, dass es sich eher um 1,3 Millionen handelt. ${ }^{7}$ Sicher scheint, dass die offiziellen Angaben der UNHCR zu registrierten Flüchtlingen in Ländern wie Jordanien und Libanon deutlich niedriger sind als die tatsächliche Anzahl der dort lebenden Flüchtlinge.

\subsection{Die Versorgungslage}

In allen drei Ländern hat der Zuzug von so vielen hilfsbedürftigen Menschen die zur Verfügung stehenden Mittel für die Grundversorgung der Bevölkerung ausgeschöpft. Das Gesundheits- und Schulwesen, die Wasser- und Nahrungsversorgung, die Versorgung mit Wohnraum usw. sind in Libanon, Jordanien und der Türkei verständlicherweise überfordert. Um die Krise zu bewältigen sind den Gastländern finanzielle und handlungsorientierte Unterstützungen aus dem Ausland sowie von internationalen Organisationen zuteilgeworden, wobei alle Regierungen und Organisationen anmerken, dass die erhaltene Hilfe nicht ausreicht. Dass dem so ist erkennt man u. a. daran, dass syrische Flüchtlinge in allen drei Aufnahmeländern zum größten Teil unterhalb der Armutsgrenze leben. In Libanon traf dies auf $76 \%$ der syrischen Flüchtlinge im Jahr 2017 zu (VASYr 2017, p. 60). In Jordanien müssen sich syrische Flüchtlinge im Prinzip in Flüchtlingslagern aufhalten. Bedingt durch das Kafilsystem ${ }^{8}$ und auch ohne amtliche Erlaubnis halten sich jedoch zirka $80 \%$ der Flüchtlinge außerhalb der Lager auf.

\footnotetext{
Libanon ist mit einer Fläche von nur $10.452 \mathrm{~km}^{2}$ nicht einmal halb so groß wie Hessen bzw. etwa so groß wie Kärnten und nicht einmal doppelt so groß wie der Kanton Wallis. Dass die tatsächliche Zahl der einheimischen Bevölkerung immer nur geschätzt werden kann beruht darauf, dass sich sowohl christliche wie auch schiitische Führer davor fürchten, dass die allenthalben bekannte Tatsache, dass Sunniten eine überwältigende Bevölkerungsmehrheit darstellen, zu einem offiziellen Fakt wird, denn politische Ämter und Sitze im Parlament werden gemäß längst überholter Bevölkerungsstatistiken paritätisch nach konfessioneller Zugehörigkeit verteilt.

6 Zahlen von November 2018, siehe https://data2.unhcr.org/en/situations/syria/location/36.

Staatsministerin für mediale Angelegenheiten, Jumana Ghunaimat, in einem Interview mit der Jordan Times vom 25. Juni 2018.

8 Ein Kafil (كفيل) ist ein Auftraggeber bzw. Patron. Im Kafilsystem sind die Angestellten sozusagen „Schützlinge“ des Kafil, also von diesem in allen Belangen abhängig, womit das im gesamten Nahen Osten übliche Kafilsystem eher einer Knechtschaft als einem rechtlich abgesicherten Arbeitsverhältnis gleicht.
} 
Von Letzteren leben 93\% unterhalb der Armutsgrenze (UNHCR 2017). In der Türkei sind Schätzungen bezüglich der Einnahmen und Ausgaben von Flüchtlingshaushalten schwieriger zu finden. Im Rahmen einer umfassenden Verwundbarkeitsstudie stellte das Welternährungsprogramm der Vereinten Nationen fest, dass ungefähr die Hälfte aller syrischen Flüchtlingshaushalte, die nicht in Lagern wohnen, ein Haushaltseinkommen aufweist, das geringer ist als die zum Überleben der Haushaltsmitglieder notwendigen Ausgaben (WFP 2018, p. 16).

In Libanon ist es syrischen Flüchtlingen nur erlaubt in drei Sektoren zu arbeiten: in der Land- und Bauwirtschaft sowie bei der Abfallentsorgung. Dies sind Sektoren, in denen Niedriglöhne die Regel sind. In Jordanien verpflichtete sich die Regierung, im Rahmen des sogenannten Jordan Compact, 200.000 Arbeitserlaubnisse an syrische Flüchtlinge zu vergeben. Als Gegenleistung erhielt Jordanien bevorzugte Handelsverbindungen zur EU sowie finanzielle Unterstützung und Investitionsversprechen. Der ehrgeizige Plan der Erteilung einer so großen Zahl an Arbeitserlaubnissen ist nicht erfüllt worden, teils weil nicht genügend Arbeitsplätze geschaffen werden konnten, teils weil man die Lebensverhältnisse und Perspektiven der syrischen Flüchtlinge nicht berücksichtigte (ODI 2018). Weiterhin ist es Flüchtlingen in Jordanien nicht erlaubt in hoch- und mittelqualifizierten Berufen zu arbeiten, weshalb viele von ihnen entweder ihren Unterhalt in der informellen Wirtschaft verdienen oder erwerbslos sind (JIF 2018, p. 9). Auch in der Türkei sind syrische Flüchtlinge in der Regel als unqualifizierte Gelegenheitsarbeiter in der Schattenwirtschaft beschäftigt, wodurch es ihnen unmöglich ist ein ausreichendes, regelmäßiges Einkommen zu erwirtschaften (FAO 2017, p. 4).

Diagramm 1: Prozentsatz syrischer Arbeiter in Libanon, die (ständig oder manchmal) arbeitsbedingten Gefahren ausgesetzt sind

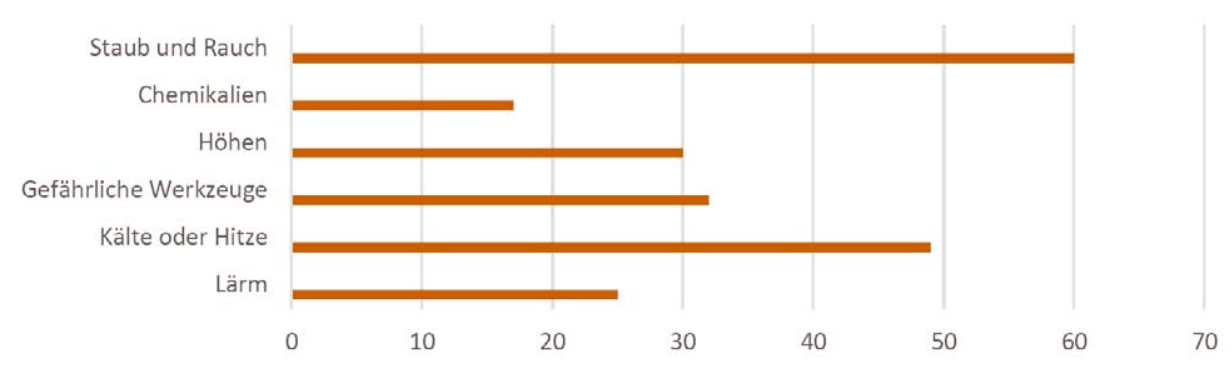

Quelle: ILO 2014, S. 32.

Die Arbeitsverhältnisse für syrische Flüchtlinge sind in allen drei Aufnahmeländern unzulänglich und oftmals gefährlich (siehe Diagramme 1 und 2). Laut eines Berichts der Internationalen Arbeitsorganisation (ILO 2014, p. 29) besitzen $92 \%$ aller 
beschäftigten syrischen Flüchtlinge in Libanon keinen Arbeitsvertrag und nur 23\% von ihnen erhalten ein regelmäßiges Monatsgehalt. Alle anderen werden stunden- oder tageweise oder auch wöchentlich und saisonabhängig angeheuert.

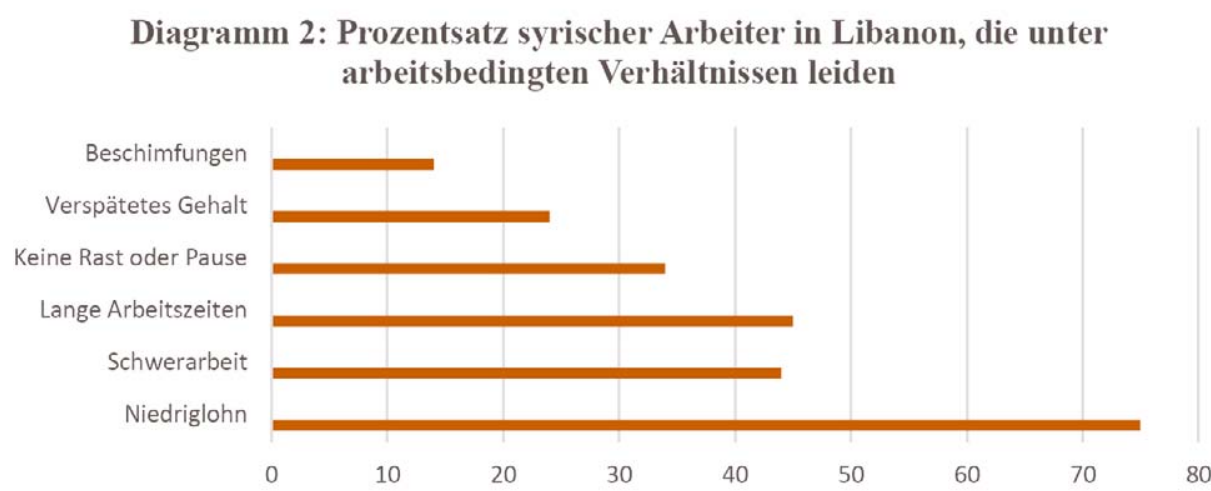

Quelle: ILO 2014, S. 32.

Für die Mehrheit der syrischen Flüchtlinge in der Türkei, Libanon und Jordanien bedeuten die unsicheren Arbeitsverhältnisse bzw. die Beschäftigungslosigkeit, dass sie von einer dauerhaften Selbstversorgung weit entfernt sind. Um zu überleben sind sie auf die Unterstützung durch Hilfsorganisationen und den öffentlichen Sektor der Aufnahmeländer angewiesen. Außerdem ist es oft notwendig sich von Verwandten, Freunden und Nachbarn Geld zu leihen. Zirka 75\% aller syrischen Flüchtlingshaushalte in Libanon haben Geld geliehen um Lebensmittel zu kaufen (VASYR 2017, p. 62) und 87\% von ihnen haben im Durchschnitt Schulden von mehr als 700 Euro (Yassin 2018, p. 45).

\subsection{Die Wohnverhältnisse}

In Libanon, Jordanien und der Türkei leben syrische Flüchtlinge in kleinen Wohnungen, Autogaragen, Rohbauten, ungenehmigten Zeltlagern, offiziellen Flüchtlingslagern usw. In Jordanien müssen, wie schon erwähnt, im Prinzip alle Flüchtlinge in einem der vier Lager wohnen, was jedoch nicht der Fall ist. Die Mehrheit hält sich in urbanen Gebieten auf, in denen Arbeit und Wohnraum verfügbar sind. Gemäß der Gesetzeslage darf dies allerdings nur, wer einen Kafil hat (siehe oben), was aber Flüchtlinge nicht davon abhält, auch ohne Kafil außerhalb der Lager zu leben. Dies führt dazu, dass syrische Flüchtlinge größtenteils ohne gültige Erlaubnis in Amman und Städten wie Mafraq, Irbid und Zarqa wohnen und arbeiten. Sollten sie von der Polizei oder anderen Ordnungskräften aufgegriffen werden, müssen sie mit einer Festnahme und Einweisung in das Flüchtlingslager bei Azraq rechnen. ${ }^{9}$ In der Türkei und in Libanon

$9 \quad$ Das Azraq-Lager ist unterbelegt und wenig beliebt, denn es liegt mitten in der Wüste, weitab von Städten und Ortschaften. 
wohnen Flüchtlinge auch zum größten Teil in den Städten. In beiden Ländern leben und arbeiten aber einige Flüchtlinge auch in ländlichen Gegenden, beispielsweise im libanesischen Bekaatal und im türkischen Süden. Syrische Flüchtlinge, die sich als Landarbeiter/innen verdingen, konkurrieren in der Türkei sowohl mit kurdischen als auch mit arabischen, Roma-, georgischen und aserbaidschanischen Arbeitsmigrant/inn/en. Von all diesen erhalten sie die niedrigsten Löhne und müssen unter den schlechtesten Bedingungen arbeiten (PELEK 2018). Die libanesische Landwirtschaft war schon immer auf Arbeitsmigrant/inn/en angewiesen. Seit den 1960er Jahren kamen diese hauptsächlich aus Syrien, wodurch die Bewohner Libanons an die Anwesenheit syrischer Landarbeiter durchaus gewöhnt waren. Die notdürftigen Unterschlupfe, üblicherweise aus Brettern und Abdeckplanen im Anschluss an einen Acker zusammengebastelt, die den männlichen Arbeitsmigranten aus Syrien vorübergehend als Obdach dienten, sind heutzutage permanente Wohnstätten für ganze syrische Flüchtlingsfamilien. In Jordanien ist die Situation ähnlich, wobei anzumerken wäre, dass man dort, anders als z. B. im fruchtbaren Bekaatal in Libanon, nur selten ungenehmigte Zeltdörfer sieht.

\subsection{Doppelte Diskriminierung}

Viele der Bretterverschläge und Zelte, die man hier und dort auf einem ungenutzten Stück Land in Jordanien sieht und die viel häufiger in Libanon und der Türkei zu finden sind, werden von nomadischen Hirten bzw. Landarbeitern und deren Familien bewohnt. Diese werden mit einem Pejorativum als „Nawar“ (نوار ; ein Wort, das möglicherweise von nar, نار, „Feuer“, abstammt) bezeichnet. Obwohl man in der wissenschaftlichen Literatur diese Bezeichnung mit gewissen Ethnien zu verbinden ${ }^{10}$ pflegt, sei anzumerken, dass die Fremdbezeichnung „Nawar“ (wie auch ähnliche regionale Bezeichnungen wie z. B. Qurbat, Qarač und Riyass) tatsächlich benutzt wird, um verschiedene prekär lebende gesellschaftliche Randgruppen zu bezeichnen. Allerdings könnte man womöglich von ,prototypischen Nawar ${ }^{\text {" }}$ sprechen. ${ }^{11}$ Das wären dann z. B. Sprecher des Domari und sogenannte Turkmenen. Jene Gruppen waren schon vor dem Krieg marginalisierte und diskriminierte Minoritäten in Syrien, indes hat sich ihre Lage im Gefolge der Flucht eher verschlechtert. Sie leiden nicht nur unter extremer Armut, sondern werden auch weiterhin von ihren syrischen Landsleuten und nunmehr zusätzlich von der aufnehmenden Bevölkerung diskriminiert. ${ }^{12}$

10 Siehe z. B. Law 2014, der mit großer Sicherheit behauptet, dass „In Syria the groups known as the Nawar include the Dom, mainly Sunni Turkmen, the Turkish-speaking and Sunni Abtal, the Quarnaqut-speaking Alban (kettle-cleaners), the Akrad and the Kaoliya" (Law 2014, p. 117).

${ }_{11}$ Im Sinne von Eleanor Roschs Prototypentheorie (siehe Rosch 1973).

12 Siehe Kirkayak Kültür o. J., Yildiz 2015 sowie Development Workshop 2016. 


\subsection{Die Problematik der Papiere}

Die Mehrheit aller syrischen Flüchtlinge in Libanon und Jordanien besitzt keine gültige Meldebestätigung bzw. Aufenthaltserlaubnis. In Libanon muss jeder syrische Flüchtling, die oder der mindestens 15 Jahre alt und nicht vom Flüchtlingskommissariat der UNO registriert ist, pro Jahr 200 USD für eine Aufenthaltserlaubnis zahlen. Da die Bearbeitung eines Antrags auf eine Aufenthaltserlaubnis bei der Behörde Monate dauern kann, kommt es vor, dass man die Aufenthaltserlaubnis erst erhält, wenn sie schon fast abgelaufen ist. Bedenkt man, dass ein syrischer Arbeiter in Libanon im Durchschnitt 200 USD im Monat verdient, müsste er $^{13}$ für jedes Familienmitglied, das 15 Jahre und älter ist, einen ganzen Monatsverdienst für eine Aufenthaltserlaubnis ausgeben. Bei einer sechsköpfigen Familie müsste er also ein halbes Jahr nur für die Aufenthaltserlaubnis der Familie arbeiten. Diese absurde Situation führt dazu, dass nur zirka ein Viertel aller syrischen Flüchtlinge in Libanon gültige Papiere besitzt.

In Jordanien ist die Lage nicht viel anders. Dort müssen sich syrische Flüchtlinge von der örtlichen Polizei eine sogenannte MOI-Karte, d. i. eine biometrische Karte des Innenministeriums, besorgen. Um solch eine Karte zu erhalten, sind syrische Flüchtlinge ab dem zwölften Lebensjahr verpflichtet ein ärztliches Attest des Gesundheitsministeriums sowie einen Mietvertrag oder eine Bestätigung des Wohnsitzes seitens des Flüchtlingslagers vorzulegen. Eine 2016 in Mafraq durchgeführte Untersuchung zeigte, dass die Hälfte aller dort wohnenden syrischen Flüchtlinge keine MOI-Karte besitzt. Auch hier scheinen Kosten und zeitraubende Behördengänge die primären Ursachen zu sein (ICMC 2017, p. 10).

In der Türkei erhalten syrische Flüchtlinge in der Regel einen vorübergehenden Schutzstatus. ${ }^{14}$ Ein Dokument, das dies bezeugt, bewahrt Flüchtlinge davor deportiert $\mathrm{zu}$ werden und berechtigt die Inhaber dazu von den Einrichtungen des Sozialstaats (Gesundheitsfürsorge, Ausbildung, Grundsicherung usw.) Gebrauch zu machen. Außerdem benötigen Flüchtlinge aus Syrien eine Arbeitserlaubnis, die jedoch die wenigsten von ihnen besitzen. Stattdessen arbeiten über eine Million syrische Flüchtlinge in der Schattenwirtschaft, die in der Türkei immerhin ungefähr ein Drittel zum türkischen Bruttoinlandsprodukt beiträgt. ${ }^{15}$

13 Nur wenige weibliche syrische Flüchtlinge verdienen Geld in Libanon, deshalb das männliche Pronomen.

14 Dies obwohl die Türkei eine von weltweit nur vier Nationen ist, die einen geographischen Vorbehalt anwendet, was bedeutet, dass sich die Türkei nur verpflichtet, die Genfer Flüchtlingskonvention von 1951 auf Flüchtlinge anzuwenden, die aus Europa kommen, wobei es in erster Linie darum ging, türkischsprachigen Flüchtlingen aus Bulgarien und dem Balkan die Anerkennung des Flüchtlingsstatus zu gewähren.

15 Schätzungen des Internationalen Währungsfonds (IMF 2018, p. 54). Ungefähr dasselbe gilt für Libanon, während die Zahlen für Jordanien widersprüchlich sind: Einerseits rechnet der Internationale Währungsfonds damit, dass nur 17,5\% des jordanischen Bruttoinlandsprodukts aus dem informellen Sektor stammt, andererseits nehmen offiziell nur 38\% der jordanischen Bevölkerung 
In allen drei Nachbarländern Syriens fehlen syrischen Flüchtlingen oftmals nicht nur Aufenthalts- bzw. Arbeitserlaubnis, sondern eine Reihe anderer wichtiger Papiere. Beispielsweise besitzen viele Flüchtlinge keine Heirats- oder Geburtsurkunde für im Nachbarland zur Welt gekommene Kinder. Ersteres lässt sich dadurch erklären, dass es unter Muslimen üblich ist religiöse Eheschließungen durch einen Scheich durchführen zu lassen, die auch den Abschluss eines Ehevertrags gemäß der Scharia beinhalten. Solche Verträge besitzen jedoch in den meisten Ländern keine gesetzliche Gültigkeit, was manchen Flüchtlingen unbekannt ist. Das basale Problem ist demzufolge die Existenz zweier paralleler legistischer Strukturen: zum einen das religiöse, ,privatrechtliche“ Regelwerk der Scharia zum anderen das Rechtssystem des jeweiligen Landes. Hinzu kommt, dass man innerhalb Syriens auch für amtliche Zwecke hauptsächlich mit dem Stammbuch auskommt. Der Verlass auf das Stammbuch kann auch als partielle Erklärung dafür dienen, dass syrische Flüchtlinge für ihre Kinder häufig keine Geburtsurkunde besitzen.

\subsection{Kontrollstellen}

Das Fehlen von „Papieren“ wird zu einem unmittelbaren, akuten Alltagsindikator für Unsicherheit, sobald man als syrischer Flüchtling an eine Kontrollstelle gelangt. Feste Kontrollstellen sind den Flüchtlingen üblicherweise bekannt, weshalb sie entweder vermeiden diese zu durchqueren oder sich Manöver ausdenken hindurchzukommen ohne entdeckt zu werden. Schwieriger ist es, plötzlich errichtete Straßensperren zu umgehen. Wer als syrischer Flüchtling ohne gültige Papiere an einem libanesischen Kontrollpunkt festgenommen wird, muss mit einer drei- bis fünftägigen Haft rechnen. Zuweilen kann die Haft aber auch bedeutend länger dauern. Die Angehörigen des Festgenommenen merken bald was geschehen ist, denn die Sicherheitskräfte schalten dessen Handy aus. ${ }^{16}$ In Jordanien werden syrische Flüchtlinge ohne gültige Papiere, wie schon erwähnt, in der Regel in das Flüchtlingslager bei Azraq eingewiesen. In der Türkei ist die Lage etwas entspannter, da die meisten Syrer im Lande einen vorübergehenden Schutzstatus genießen. Dennoch geht aus unseren Gesprächen mit syrischen Flüchtlingen hervor, dass Kontrollstellen generell als Risiken aufgefasst werden, was, wie im folgenden Abschnitt deutlich werden wird, in erheblich höherem Maß für Syrien gilt.

am Arbeitsmarkt teil, was weltweit der niedrigste Prozentsatz wäre (JRP 2018, p. 28). Es ist anzunehmen, dass viele Jordanier im informellen Sektor beschäftigt sind.

16 Die maskuline Form ist in diesem Zusammenhang angebracht, denn die Sicherheitskräfte der Nachbarländer Syriens konzentrieren sich bei ihren Kontrollen auf männliche syrische Flüchtlinge. 


\section{Die Lage in Syrien}

\subsection{Die politische Situation}

Der Krieg in Syrien hat Tod und Zerstörung gebracht. Es ist belegt, dass alle Konfliktparteien Kriegsverbrechen begangen haben. Allerdings ist das schiere Ausmaß der Verbrechen, dessen sich das Regime ${ }^{17}$ unter Baschar Assad schuldig gemacht hat, ungleich viel größer als das der übrigen Kriegsparteien. Die Folter und Ermordung von Zehntausenden Inhaftierten, das Abwerfen von mehr als 70.000 Fassbomben und die Anwendung von chemischen Waffen gegen die eigene Bevölkerung sind nur einige der Verbrechen, die das syrische Regime begangen hat. Nur durch die militärische Unterstützung durch Russland und den Iran ist es dem Assad-Regime gelungen den Krieg zu gewinnen und weiterhin an der Macht zu bleiben. Der Machterhalt des Regimes ist der alles überragende Indikator für Unsicherheit in Syrien, denn Millionen von Syrern ist es nicht möglich in ihr Land zurückzukehren, da sie dort unter Umständen Gefangenschaft, Folter und Tod erwartet. Es ist allenthalben bekannt, dass das Regime Fahndungslisten benutzt, auf denen die Namen von Millionen syrischer Staatsbürger verzeichnet sind. Wer an oppositionellen Demonstrationen teilgenommen oder sich in alten bzw. neuen Medien gegen das Regime ausgesprochen hat, wer regimekritische Verwandte hat oder aus ehemals von der Opposition kontrollierten Gebieten kommt, wer die Einberufung zum Wehrdienst vermieden hat oder desertiert ist etc., dessen bzw. deren Namen ist mit großer Wahrscheinlichkeit auf einer der Fahndungslisten eingetragen. Dazu sagte Jamil Hassan, Leiter des syrischen Luftwaffengeheimdienstes und Berater von Baschar Assad, für den der deutsche Bundesgerichtshof wegen Verbrechen gegen die Menschlichkeit einen internationalen Haftbefehl ausgestellt hat, bei einem Treffen mit seinen Geheimdienstkollegen: „Ein Syrien mit zehn Millionen vertrauenswürdigen Leuten, die der Führung gehorchen, ist besser als ein Syrien mit dreißig Millionen Wandalen. " 18 Im Klartext bedeutet das, dass die Flucht und Vertreibung von Millionen syrischer Staatsbürger für das Regime eine Gelegenheit zur politischen Säuberung bietet. Bei allen Verhandlungen um die Rückkehr von Flüchtlingen nach Syrien gilt es nicht zu übersehen, dass in Syrien nach wie vor eine Diktatur herrscht, in der Bürger- und Menschenrechte kaum geachtet werden und dass sich das syrische Regime aussuchen wird, wen es ins Land zurückkommen lassen wird.

\footnotetext{
17 Wir gebrauchen den Ausdruck „Regime“ als Übersetzung des arabischen Begriffs Nizam (نظام), was so viel wie ,das System“” oder „die Ordnung“ bedeutet und die gängige Bezeichnung für das jeweilige politische Regime ist.

18 Vgl. $<$ https://syrianobserver.com/EN/features/19769/jamil_hassan_any_all_opposition_will _be_eliminated.html>
} 


\subsection{Rückkehr?}

Das russische und das syrische Regime bemühen sich momentan, die Rückkehr aus der Flucht als sicher und zukunftsträchtig darzustellen. Dem widersprechen Zeugenberichte über in Haft genommene, getötete und verschwundene Rückkehrer. ${ }^{19}$ Im Alltag sind es, ähnlich wie in den Nachbarländern, die Kontrollstellen in Syrien, die Furcht einjagen. Allerdings ist eine Festnahme an einer syrischen Grenz- oder Binnenkontrolle deutlich gefährlicher als an einem libanesischen, jordanischen oder türkischen Kontrollpunkt. Statt vorübergehender Haft oder Einweisung in ein Flüchtlingslager kann eine Festnahme in Syrien sehr wohl mit Folter und/oder Tod enden. ${ }^{20}$ Hinzu kommt, dass viele der angeblich unter Regierungskontrolle stehenden Gebiete Syriens gar nicht vom syrischen Militär beherrscht werden, sondern von ausländischen Streitkräften wie Hisbollah und privaten Sicherheitsunternehmen (wie der russischen Gruppe Wagner), örtlichen Clanmitgliedern und regelrechten Gangsterbanden (wie den berüchtigten Schabiha-Milizen, an deren Spitze zwei Cousins Baschar Assads stehen). Zu unterscheiden zu welcher Gruppierung die bewaffneten Männer gehören, die eine Kontrollstelle bemannen, ist nicht immer leicht. Uniformen, Insignien, gehisste Flaggen usw. können mitunter zu Täuschungszwecken eingesetzt werden. Wenn bei einer Kontrolle vordergründig unverfängliche Fragen gestellt werden, wie „Wohin geht die Fahrt?“ oder „Woher kommt ihr?“", gilt es richtig zu raten und eine Antwort zu geben, die den bewaffneten Männern recht ist. Da Syrien kein Rechtsstaat ist, herrscht ohnehin Willkür und jede Kontrollstelle wird somit zu einer unmittelbaren Bedrohung. Erwägungen bezüglich einer Rückkehr sind gemäß den Aussagen unserer syrischen Gesprächspartner von erheblicher Unsicherheit (khatar; خطر) geprägt.

\subsection{Umfragen zu den Absichten syrischer Flüchtlinge}

UNHCR und die Carnegie Endowment for International Peace haben syrische Flüchtlinge über ihre Absichten in Bezug auf eine eventuelle Rückkehr nach Syrien befragt. ${ }^{21}$ Die Umfragen wurden telefonisch an Wochenenden durchgeführt und sammelten in erster Linie quantifizierbare Daten ein. Aus den Berichten, die zu den Umfragen erstellt wurden, geht hervor, dass die überwältigende Mehrheit der Befragten nicht beabsichtigt in den nächsten sechs bzw. zwölf Monaten nach Syrien zurückzukehren. Unsicherheit wird als primärer Grund für diese Entscheidung angegeben. Dazu haben wir in unmittelbaren Begegnungen mit syrischen Flüchtlingen im Rahmen von Fokusgruppen, die wir zumeist in den Unterkünften der Flüchtlinge durchgeführt haben, qua-

\footnotetext{
19 Solche Berichte wurden einerseits von syrischen Flüchtlingen in unseren Fokusgruppen vorgetragen und andererseits vom libanesischen Minister für Flüchtlingsfragen Mouin Merhebi veröffentlicht.

20 Auch hierzu gab es Aussagen syrischer Flüchtlinge in unseren Fokusgruppen (siehe weiter unten).

${ }_{21}$ UNHCR 2018a und 2018b, sowie Carnegie Endowment for International Peace 2018.
} 
litative Daten zusammengetragen, die wir nun anhand von Zitaten und Kommentaren vorstellen möchten.

\section{Mit eigenen Worten}

Wenn syrische Flüchtlinge in unseren Diskussionen über die Zukunft sprachen, bezogen sie sich fast immer auf die Vergangenheit, d. h. auf die Zeit vor dem Krieg. Was zum Ausdruck kam, war ein Gefühl des Verlustes. Eine Frau, die schon seit sechs Jahren in Libanon lebte, meinte: ,Wir wünschen, dass wir wieder zurück in unser Zuhause könnten und so leben könnten wie früher. "Eine andere Frau machte die Verflechtung von Vergangenheit und Verlust anschaulich: „Ich bewahre immer noch den Schlüssel zu meinem Haus auf, obwohl ich weiß, dass es dem Erdboden gleichgemacht wurde. “ Bildlich gesprochen warten syrische Flüchtlinge, den Schlüssel zu dem, was verloren gegangen ist, in der Hand haltend, auf Gestern. ${ }^{22}$

\subsection{Analytische Kategorien}

Angesichts der prekären Situation, in der sich die meisten syrischen Flüchtlinge befinden, ist zu erwarten, dass es ihnen nicht schwer fällt Alltagsindikatoren für Unsicherheit zu entdecken. Dabei sind unmittelbare Indikatoren, wie z. B. körperliche und verbale Angriffe, mit strukturellen Indikatoren, wie Armut, „Illegalität“ und Rassismus, kausal verbunden, denn die strukturbedingte Schutzlosigkeit und Verletzlichkeit der Flüchtlinge macht An- und Übergriffe auf sie erst möglich. Alltagsindikatoren für Unsicherheit werden nicht nur durch eigene Erfahrungen sichtbar, sie sind auch Gegenstand des Informationsaustausches der Syrer untereinander zur Sicherheitslage in den Aufnahmeländern bzw. in Syrien. Diese Sicherheitskommunikation wird wiederum zum Ausgangspunkt für Entscheidungen bezüglich des Verbleibens im Aufnahmeland oder die Rückkehr nach Syrien. Alltagsindikatoren für Unsicherheit, Sicherheitskommunikation und Absichten der syrischen Flüchtlinge sind somit logisch miteinander verkettet.

\subsection{Alltagsindikatoren für Unsicherheit}

Im Folgenden nun sollen Zitate aus den Fokusgruppen mit syrischen Flüchtlingen einen Einblick in die Sicherheitslage im Libanon geben:

„Libanesische Jungen schikanieren und demütigen uns auf der Straße und wir müssen das alles hinnehmen. Wir können uns nicht an das Gesetz wenden, weil

$22 \quad$ Mit Bezug auf Ernst Bloch (1985) könnte man sagen, dass sich die Flüchtlinge in ihren Hoffnungen eher auf ein Nicht-Mehr-Sein als auf ein Noch-Nicht-Sein beziehen. 
wir ohne Genehmigung nach Libanon gekommen sind und keine Papiere haben. " (Älterer Mann, ursprünglich aus Rif Homs) ${ }^{23}$

„Hier [in Libanon] fürchten wir die Rowdys (Az’ar; أز عر). Als syrischer Mann kann ich nicht einmal meine eigene Schwester vor sexuellen Belästigungen durch Libanesen schützen. Man sieht sogar wie die Offiziere der libanesischen Polizei mit den Rowdys zusammenstehen und sich unterhalten. Sogar die Polizisten können nichts gegen sie unternehmen. " (Mann, ursprünglich aus Rif Aleppo)

„Jedes Mal, wenn etwas gestohlen wird, ein Auto oder Fernseher, gehen wir nicht raus, weil jeder annimmt, dass ein Syrer das gemacht hat. " (Frau, ursprünglich aus Homs)

„Eines Abends haben mich bewaffnete Männer festgenommen. Sie haben mich gefragt, was ich hier in Libanon zu suchen habe und warum ich nicht zurückgehe nach Syrien um zu kämpfen. Sie haben mich verhört und geschlagen. Du bist eine Zielscheibe für Demütigungen, wenn du gebildet bist. " (Ehemaliger Student, ursprünglich aus Idlib)

„,Einige Männer haben meinen Schwager angerufen und ihm Arbeit angeboten. Er ist zur vereinbarten Zeit und Stelle gegangen, aber stattdessen haben Männer ihn gefesselt und vier Stunden lang verprügelt. Sie haben seine Nase gebrochen. Sie haben ihn beschuldigt, dass er Mitglied von IS oder der Nusra-Front ist. " (Frau, ursprünglich aus Dar'a)

„Um acht Uhr abends kamen Männer, die wahrscheinlich zur schiitischen AmalBewegung gehören, in meine Wohnung und verlangten mein Handy und meine Papiere zu kontrollieren. Sie fragten mich über mich und meine Familie aus. Sogar in meiner eigenen Wohnung kann ich mich nicht sicher fühlen. " (Junger Mann, ursprünglich aus Hasaka)

In den Teilen Libanons in denen die Hisbollah herrscht müssen syrische Flüchtlinge einmal im Jahr ein detailliertes Formular zur Volkszählung ausfüllen. Manchmal führen Männer, die behaupten, dass sie zur Hisbollah gehören, Razzien auf Arbeitsplätzen durch. Diese Männer „konfiszieren“ das Geld, das syrische Flüchtlinge bei sich haben. Die libanesischen Sicherheitskräfte führen ebenfalls Razzien durch:

„,Die Soldaten kommen im Winter um fünf Uhr morgens. Sie holen alle Männer aus den Zelten und stellen sie in einer Reihe auf. Dann kontrollieren sie die Namen von jedem. Sie wollen uns einschüchtern. " (Älterer Mann, ursprünglich aus Rif Homs)

23 Das Wort Rif(ريف) bezeichnet die ländliche Umgebung einer Stadt oder eines Ortes. 
„, Wir sind nach Libanon gekommen, um uns sicher zu fühlen. Stattdessen sind wir hier auch unsicher. Soldaten brechen unsere Türen auf und kommen in unsere Wohnungen. Der Unterschied ist, dass die Soldaten in Syrien hereinstürmen und Mädchen vergewaltigen, die Männer demütigen und sie mitnehmen oder umbringen. “ (Mann mittleren Alters, ursprünglich aus Rif Homs)

Wie oben erwähnt, sind Sicherheitskontrollen (Checkpoints) ein wichtiger Alltagsindikator für Unsicherheit:

„Jeden Tag haben wir Angst, dass wir an den Kontrollstellen festgenommen werden. " (Mann mittleren Alters, ursprünglich aus Rif Homs)

„,Wenn das Handy der Männer ausgeschaltet ist, wissen wir, dass sie an einer Kontrollstelle festgenommen wurden und dass es mindestens drei Tage dauern wird, bis sie wieder freikommen. “ (Ältere Frau, ursprünglich aus Qusair)

„An einer Kontrollstelle in den schiitischen Vororten im Süden von Beirut haben sie meine Papiere beschlagnahmt. Ich versuche schon seit drei Jahren sie wieder zurückzubekommen! Keiner kann mir helfen, wenn ich die Informationsstelle der UNO anrufe. " (Ältere Frau, ursprünglich aus Ghuta)

„, Wenn sie mich aus der Haft entlassen, sagen die Soldaten mir, dass ich meine Papiere erneuern muss. Sie geben mir ein Papier, das es mir erlaubt mich 15 Tage lang frei zu bewegen, damit ich zur Polizei gehen kann, um meine Papiere zu erneuern, obwohl sie wissen, dass das nicht geht. Es ist unmöglich. " (Junger Mann, ursprünglich aus Rif Homs)

Im Anschluss nun Zitate zur Sicherheitslage in Syrien, die ebenfalls aus den Fokusgruppen mit syrischen Flüchtlingen stammen:

„, In Syrien ist jeder Checkpoint verschieden. Alle zwanzig Meter kommt eine Kontrollstelle und man weiß nicht, auf wen man da trifft. “ (Junge Frau, ursprünglich aus Hasaka)

„, Mein Bruder lebt immer noch in Rif Homs. Er hat Felder außerhalb des Dorfes und fuhr mit einem Minibus, um nach ihnen zu schauen. An einem Kontrollpunkt des [syrischen] Regimes haben sie alle Ausweise kontrolliert und alle Männer im Bus festgenommen. Mein Bruder ist im Dorf X registriert, so steht es in seinem Ausweis, aber er hat sein ganzes Leben lang im Dorf Y gewohnt. Alle Männer im Bus waren aus dem Dorf X. Die Soldaten haben die Männer in eine ehema- 
lige Lagerhalle einer Fabrik gebracht. Mein Bruder hat die Soldaten stundenlang angefleht, bis sie ihm geglaubt haben und ihn haben gehen lassen. Er weiß nicht was mit den anderen Männern passiert ist. " (Mann mittleren Alters, ursprünglich aus Rif Homs)

„, Mein Schwager in Syrien hat Verwandte in einem anderen Dorf besucht. Er hat die Kontrollstelle durchquert und man hat ihm versichert, dass für ihn alles in Ordnung ist. Zwei Tage später kam er zur selben Kontrollstelle und wurde beiseite genommen. Er wurde gegen seinen Willen zum Wehrdienst verpflichtet und 28 Tage später ist er in Deir ez-Zor gefallen. " (Frau mittleren Alters, ursprünglich aus Ghuta)

Gemäß Aussagen einiger syrischer Flüchtlinge ist es mitunter möglich Soldaten in Syrien zu bestechen, so dass man unbehindert Grenzposten und Kontrollstellen durchqueren kann, wie folgendes Zitat beweist:

„Ich musste mich operieren lassen, was in Libanon 1.600 USD gekostet hätte. Ich bin zurück nach Aleppo gefahren und habe dort die Operation umsonst gemacht. Ich kam durch, weil ich meinen Geburtstag in allen Unterlagen vier Jahre früher als er wirklich ist angegeben habe. Auf dem Papier bin ich 17, nicht 21 [also noch zu jung, um zum Wehrdienst eingezogen zu werden]. Ich habe gesehen, dass alles wieder einigermaßen normal ist in Aleppo, obwohl natürlich alles vom Krieg gezeichnet ist. Was mir aufgefallen ist, sind die vielen fremden Soldaten: Russen, Perser und Hisbollah. Überall! Ich hatte einen kleinen Zettel, der von einem syrischen Grenzsoldaten unterschrieben war [hält ein Stück Papier, das von einem größeren Blatt abgerissen wurde, hoch] auf dem stand, dass ich alle Kontrollen in Aleppo passieren darf. Ich habe dafür 5.000 syrische Lira [ungefähr 100 USD] gezahlt und es hat funktioniert! “ (Junger Mann, ursprünglich aus Aleppo)

Immer wieder wurde der in den Aufnahmeländern wesentliche Alltagsindikator der Armut von den Teilnehmern in den Fokusgruppen angesprochen:

„Auch wenn man in Syrien arm war, war man nie so arm wie hier in Libanon." (Junge Frau, ursprünglich aus Ghuta)

„,Die Libanesen erzählen uns, dass sie uns beherbergen. Aber in Wirklichkeit zahlen wir selber für alles. Wir zahlen Miete, wir zahlen für medizinische Behandlungen und wir werden ausgebeutet, wenn wir für sie arbeiten. " (Junger Mann, ursprünglich aus Rif Hama) 
„Libanesen nehmen uns unser Geld weg, indem sie vorgeben von der Hilfe [Ighatha; إغاثة, ein Kürzel, das für die Summe aller Hilfsorganisationen benutzt wird] zu sein, damit sie an unsere Personendaten herankommen. Wir bekommen SMS vom Roten Kreuz und von der UNO, in denen sie uns davor warnen, dass Leute kommen können, die behaupten, von ihnen zu sein. Sie schreiben: Seid vorsichtig! “ (Junger Mann, ursprünglich aus Rif Homs)

,,Jemand benutzte drei Jahre lang meine Taghzieh-Karte [تغزبيه; eine Zahlkarte der UNO, mit der man das Notwendigste kaufen kann]. Ich bin eine Witwe mit drei kleinen Kindern. Ich ging immer wieder zur UNO, um mich zu beschweren, aber die haben immer geantwortet, dass ich registriert bin und dass meine Karte aktiviert ist. Aber ich hatte doch gar keine Karte! Ein Anwalt von einer Hilfsorganisation hörte mir zu, als er unser Lager besuchte und kümmerte sich um meinen Fall. Es stellte sich heraus, dass ein libanesischer Mann meine Karte benutzte. Der Anwalt organisierte ein Treffen mit dem Mann, der sich mir gegenüber entschuldigte und erklärte, dass er es kaum schafft, seine fünfköpfige Familie zu ernähren. Was sollte ich tun? Ich habe ihm vergeben. Aber es macht viel aus, endlich meine Karte zu haben. " (Frau mittleren Alters, ursprünglich aus Rif Aleppo)

„, Taxifahrer verlangen mehr Geld von Syrern, wenn sie sich einer Kontrollstelle nähern. “ (Älterer Mann, ursprünglich aus Homs)

„,Die UNO konzentriert sich nicht auf die Bedürfnisse der Flüchtlinge. Ich habe gesehen, wie die UNO 10.000 USD für einen Workshop ausgibt, während Leute Essbares aus dem Müll sammeln. " (Syrischer Freiwilliger, der in einem der palästinensischen Flüchtlingslager in Libanon arbeitet)

\subsection{Sicherheitskommunikation}

Syrische Flüchtlinge benutzen alle verfügbaren Medien, um Auskünfte und Nachrichten über die Geschehnisse in Syrien zu sammeln. Sie sehen sich verschiedene Fernsehkanäle an, forschen im Netz nach, kommunizieren mit Hilfe sozialer Medien usw. Jedoch ist ihr Informationssuch- und Kommunikationsverhalten von einem allgemeinen und grundsätzlichen Misstrauen gegenüber allen Quellen geprägt:

„, Wir wissen sehr wohl, wann die Nachrichten lügen. Durch die sozialen Medien gibt es keine Geheimnisse mehr. Wenn wir hören, dass ,, Terroristen “ oder ,, bewaffnete Gruppen " ein Gebiet angefallen haben, wissen wir sofort, dass das eine Lüge ist, weil die oppositionellen Gruppen gar nicht die richtigen Waffen besitzen, um das Gebiet zu beschießen. (Mann, ursprünglich aus Homs) 
„Ich schaue den [regimetreuen] Addounia-Kanal im Fernsehen an und ganz egal, was die behaupten, drehe ich die Information herum. Wenn die behaupten, dass „,terroristische Banden“ diese oder jene Stadt angefallen haben, weiß ich, dass es wahrscheinlich die syrische Armee war, die das gemacht hat. " (Frau, ursprünglich aus Damaskus)

Syrische Flüchtlinge gaben an, dass sie folgende Fernsehkanäle anschauen: Al Jadeed (Libanon), Al Jazeera (Qatar), Al Arabiya (Emirate) und Al Hadath (Emirate). Alle Teilnehmer an den Fokusgruppen berichteten, dass sie auch den regimetreuen syrischen Fernsehsender Addounia TV anschauen, obwohl sie diesem nicht vertrauen. Es vollzieht sich auch ein elektronischer Informationsaustausch über die syrische Grenze mit Hilfe von sozialen Medien wie WhatsApp und Facebook. Diese Kommunikation geschieht jedoch im Schatten der Überwachung durch die syrischen Geheimdienste (zusammenfassend Mukhabarat, مخابر ات, genannt). Auch die Geheimdienste der Aufnahmeländer scheinen diese Kommunikation zu überwachen.

„Hier in Libanon bekam ich einen Anruf von einer Frau, die für den [libanesischen] militärischen Geheimdienst arbeitet. Sie kannte viele Einzelheiten über mich und meine Familie. Nach diesem Anruf habe ich aufgehört mit meiner Familie am Handy Einzelnes zu besprechen." (Witwe mittleren Alters, ursprünglich aus Homs)

,, Mein Vater hat aus Versehen den Gefällt-mir-Knopf zu einem IS-Video gedrückt, als ich auf Facebook war. Ich bekam einen Anruf vom libanesischen Mukhabarat: Ich sollte zu ihnen kommen und mein Handy mitbringen. Ich bin hingegangen und habe erklärt, dass es ein Versehen war. Als sie mir nicht glaubten, habe ich ihnen erzählt, dass mein Bruder bei der syrischen Armee ist. Es stellte sich heraus, dass einer der Geheimdienstoffiziere meinen Bruder kannte. Er zeigte mir sogar Bilder von ihm. Ich hatte meinen Bruder schon seit Jahren nicht mehr gesehen. " (Junger Mann, ursprünglich aus Rif Aleppo)

,Wir sind vorsichtig damit, was wir hier in Libanon sagen. Klar, wir sind in einem anderen Land, aber es ist ein Land, das mit dem syrischen Regime freundschaftlich verbunden ist. " (Junge Frau, ursprünglich aus Idlib)

Mit Freunden, Verwandten und anderen Kontakten in Syrien zu kommunizieren wird von den Flüchtlingen als gefährlich eingestuft. Einer Person, die sich in Syrien befindet, direkte Fragen zur Lage zu stellen ist risikobehaftet denn, ganz egal was sie antwortet, kann dies zu ihrem Nachteil ausgelegt werden. Deshalb vermeiden Syrer, die in Syrien leben, auf Fragen, die irgendwelchen Verdacht erwecken könnten, zu antworten. Das tun sie, indem sie entweder das Thema wechseln oder einfach auflegen. 
Oder aber man kommuniziert mit Hilfe von Verschlüsselungen. „Es regnet“ bedeutet dann, dass man unter Beschuss ist. „Es ist sehr staubig“ deutet an, dass viel Staub durch Beschuss aufgewirbelt wird. Der Satz „Es ist sehr heiß“ lässt darauf schließen, dass in der Nähe hitzige Kämpfe stattfinden. So ist auch der Satz „Nebenan findet eine Hochzeit statt" zu verstehen (denn syrische Hochzeiten sind üblicherweise mit viel Krach verbunden und zum Zelebrieren werden außerdem Schüsse abgefeuert). „Er besucht das Haus seiner Tante" bedeutet, dass jemand von den syrischen Sicherheitsorganen festgenommen wurde. Und so fort.

Abgesehen von sozialen Medien gibt es andere Möglichkeiten Informationen über die Grenze zu tragen. So kann man z. B. Fahrer von Taxis und LKWs bezahlen, um in Syrien Informationen zu sammeln und an Flüchtlinge in den angrenzenden Ländern weiterzugeben. Weibliche syrische Flüchtlinge können leichter als Männer die Grenze nach Syrien überqueren, riskieren aber dabei sexuelle Belästigungen durch Männer in Uniform. Frauen haben deshalb Methoden entwickelt, um dem vorzubeugen:

„Frauen die zurück nach Syrien gehen schmieren sich mit Schmutz ein und waschen sich mehrere Tage lang nicht, so dass sie übel riechen und die Soldaten sich von ihnen fernhalten. " (Älterer Mann, ursprünglich aus Homs)

\subsection{Qualitative Absichtsdaten}

Für syrische Flüchtlinge ist die Frage der Rückkehr überaus komplex, denn sie ist beeinflusst von den Fluchterfahrungen, der Kommunikation über die Lage in Syrien, den Push-Faktoren im Aufnahmeland, der Einschätzung wie sich die Lage in Syrien und den Nachbarländern entwickeln wird, sowie, in einigen Fällen, von politischen Überzeugungen.

„Syrien zu verlassen war traumatisch. Wir sind von sechs Uhr abends bis sechs Uhr in der Früh gewandert. Die Männer liefen voraus, falls es zu Schießereien kommen würde. Sie trugen Waffen, um uns zu verteidigen. Die Frauen und Kinder liefen weiter hinten. Wir mussten uns den Weg heraus aus Syrien erkämpfen, denn wir wurden von Regimesoldaten angegriffen, nachdem wir von einem alawitischen Dorf verraten wurden. Es kam zum Schusswechsel. " (Junger Mann, ursprünglich aus den Bergen in Rif Homs)

„Mir wurde gesagt, dass mein Mann tot ist. Ich wartete nicht ab ihn zu sehen oder ihn zu begraben, sondern packte meine Kinder, stieg in ein Taxi ein und fuhr nach Libanon. " (Frau mittleren Alters, ursprünglich aus Homs) 
„,Mein Bruder sagte mir, dass ich einem Unbekannten Geld geben sollte. Ich würde ihn zu verabredeter Zeit in einer bestimmten Straße treffen. Es war sicherer für mich als für meinen Bruder herauszugehen. Frauen wecken nicht so viel Verdacht wie Männer. Ein Soldat des Regimes entdeckte mich aber und nahm mich mit zur Station. Ich wurde verhört und man befahl mir Geschichten darüber zu bestätigen, was die Salafisten den Frauen und Kindern an schlimmen Sachen antun. Es waren furchtbare Geschichten und ich sagte, dass das soweit ich weiß nicht stimmt. Die Soldaten behandelten mich schlecht und schlugen mich. Dann wurde ihnen mitgeteilt, dass sie von meinem Bruder bestochen worden waren. Plötzlich änderte sich ihr Verhalten um 180 Grad und sie entschuldigten sich. Einer der Soldaten gab mir seine Nummer, falls ich später noch Hilfe brauchte. " (Ältere Frau, ursprünglich aus Qusair)

„, Mein Schwager war sieben Monate lang verschwunden. Seine Verwandten wurden vom Mukhabarat gerufen. Sie sollten seinen Ausweis abholen. Dann sind sie auch verschwunden. " (Frau mittleren Alters, ursprünglich aus Rif Hama)

„, Meine Schwester und ich saßen nebeneinander im Taxi, als wir angehalten wurden und sie festgenommen wurde. Ich traute mich nicht zu sagen, dass ich ihre Schwester bin. Sonst hätten sie mich auch noch mitgenommen." (Junge Frau, ursprünglich aus Homs)

„, Mein Sohn war fünf Jahre alt, als der Krieg anfing. Jedes Mal, wenn eine Rakete in ein Gebäude einschlug, hat er in die Hosen gemacht. Eines Tages wurde unser Balkon getroffen. Das war der Wendepunkt für mich. Ich hatte Angst, dass ich meine Kinder verlieren würde. Wir sind nach Libanon geflohen. Jetzt können wir nicht zurück nach Syrien, weil zwei meiner Söhne alt genug sind, zur syrischen Armee einberufen zu werden. " (Frau mittleren Alters, ursprünglich aus Qunaitra)

Die Frage der Rückkehr hat letztendlich auch mit dem Vertrauen in die Zukunft Syriens zu tun. Da Syrien schon seit Jahrzehnten eine Diktatur ist, kann man davon ausgehen, dass die meisten Syrer dem Regime wenig Vertrauen entgegenbringen. Allerdings scheint der Vertrauensmangel auch auf den sozialen Zusammenhalt überhaupt übergegriffen zu haben:

„Es stellt sich heraus, dass dieselbe Person, die uns mit Brennstoff und Essen versorgt hat, als wir in Baba Amr belagert wurden, ein Spion für das Regime war. Wem können wir überhaupt noch vertrauen? " (Frau, ursprünglich aus Baba Amr)

Diese Frage - „Wem können wir überhaupt noch vertrauen?“ - hat entscheidende Folgen für die Absichten syrischer Flüchtlinge. Das tiefe und weit verbreitete Misstrauen bedeutet, dass man offiziellen Garantien und Amnestien, auch wenn sie von inter- 
nationalen Organisationen unterschrieben würden, mit größter Skepsis begegnen wird. Hinzu kommt, dass der Krieg sogar die engsten sozialen Beziehungen zerrüttet hat:

„,Wenn meine Familie mir erzählt, dass es sicher ist nach Syrien zurückzukehren, werde ich ihnen nicht glauben. Sie werden vielleicht vom Regime gezwungen, das zu sagen. " (Mann mittleren Alters, ursprünglich aus Homs)

Der Krieg scheint eine solidarische Gesellschaft, geschweige denn eine Gemeinschaft, unmöglich gemacht zu haben:

„Wie sollen wir denn mit und neben denen leben, die uns betrogen und getötet haben? Es wird kein Zusammenleben wie vorher geben. " (Älterer Mann, ursprünglich aus Homs)

„Nichts ist wie vorher. Die Leute sind anders. Die Städte sind anders. Alles ist anders. Du kennst keine der Leute mehr. " (Junge Frau, ursprünglich aus Aleppo)

„, Syrien wird zu Libanon [d. h. Syrien wird durch konfessionelle Zugehörigkeiten und Konflikte zersplittert]. “ (Junge Frau, ursprünglich aus Damaskus)

„Familien, die in den Dörfern geblieben sind, nehmen sich die Häuser von denen, die gegangen sind, und verkaufen sie und verdienen damit Geld. Soldaten und Kämpfer bringen ihre Familien und besetzen leerstehende Häuser und bestellen das Land. Bilde dir nicht ein, dass man die wieder wegbekommt. " (Frau, ursprünglich aus Rif Qusair)

„Die UNO arbeitet mit den libanesischen Sicherheitsorganen zusammen und die libanesischen Sicherheitsorgane arbeiten mit dem syrischen Regime zusammen." (Mann mittleren Alters, ursprünglich aus Damaskus)

\section{Alltagsindikatoren, Kommunikation, Absichten und dauerhafte Lösungen}

Wie eingangs erwähnt, schlägt die UNO drei dauerhafte Lösungen für Flüchtlinge vor:

1. freiwillige Rückkehr in Sicherheit und Würde,

2. Resettlement in ein Drittland oder

3. die Integration im Aufnahmeland. 
Wie aus den Zitaten aus unseren Fokusgruppendiskussionen hervorgeht, stimmt die erste Lösung, nämlich die freiwillige Rückkehr, wenig mit den Absichten der syrischen Flüchtlinge, mit denen wir gesprochen haben, überein. Zum Resettlement in ein Drittland, also in ein Land, das weder Syrien heißt noch ein Anrainerstaat Syriens ist, sondern eine sichere, zukunftsträchtige Destination im sogenannten globalen Norden ist, wäre zu sagen, dass sich manche Flüchtlinge, mit denen wir gesprochen haben, durchaus wünschen würden in ein solches Drittland überzusiedeln. Es ist jedoch wichtig zu betonen, dass dies für die Mehrzahl der Teilnehmer in unseren Fokusgruppen keine Lösung darstellt. Einigen der befragten Personen wurde beispielsweise das Resettlement nach Europa bzw. Nordamerika angeboten, was diese aber ablehnten. Als Begründung gaben sie auf unsere Fragen an, dass sie befürchten würden, ihre Kinder zu verlieren:

„Die UNO erzählte uns, dass unsere Töchter das Kopftuch ablegen müssen und dass sie am Schwimmunterricht mit Jungen teilnehmen müssen. " (Zwei Frauen mittleren Alters, beide ursprünglich aus Homs)

„In Europa nehmen sie dir die Kinder weg, wenn du sie züchtigst. Sie akzeptieren auch Sex vor der Ehe. " (Frau mittleren Alters, ursprünglich aus Homs)

Obwohl diese Kommentare nicht typisch für die Auffassung von syrischen Flüchtlingen sind, werden sie hier angeführt, um den Mangel an kommunikativer Arbeit von offizieller Seite aufzudecken. Dass Resettlement für die Mehrheit der syrischen Flüchtlinge keine Lösung darstellt, hat aber weniger mit Missverständnissen als mit einer realistischen Einschätzung der eigenen Lage zu tun. Für die meisten syrischen Flüchtlinge, mit denen wir gesprochen haben, bedeutet das Verbleiben in der Nähe Syriens, dass man einerseits die Tür für eine eventuelle Rückkehr offenhält und dass man andererseits, zumindest in Libanon und Jordanien, die Sprache beherrscht und mit den sozialen Normen vertraut ist. Da eine Rückkehr, wenn überhaupt, hinter dem Horizont der überschaubaren Zukunft liegt, gilt es für die Frage der Integration im Aufnahmeland - also vor allem in der Türkei, im Libanon und in Jordanien - eine befriedigende Antwort zu finden. Die politische Brisanz dieser Frage sollte nicht dazu verleiten, dass sie unter den Teppich gekehrt wird, denn die Dauerhaftigkeit der Integrationsfrage hängt nicht zuletzt von der Dauerhaftigkeit der Flucht ab. Und diese hängt wiederum im Wesentlichen vom Verbleib des syrischen Regimes ab. 


\section{Bibliographie}

Bloch, E. (1985), Werkausgabe, Band 5: Das Prinzip Hoffnung. Frankfurt am Main, Suhrkamp.

Carnegie Endowment for International Peace (ed.) (2018), Unheard Voices: What Syrian Refugees Need to Return Home. Beirut, Carnegie Endowment for International Peace.

Development Workshop (2016), Living at the Bottom: Dom Migrants from Syria-On the Road Amid Poverty and Discrimination. Ankara, Development Workshop.

FAO (ed.) (2017), Turkey: Syrian Refugee Resilience Plan, 2017-2018. Ankara, Food and Agriculture Organization of the United Nations.

ICMC (ed.) (2017), Undocumented, Unseen, and At Risk: The Situation of Syrian Refugees Lacking Civil and Legal Documentation in Jordan. Geneva, International Catholic Migration Committee.

ILO (ed.) (2014), Assessment of the Impact of Syrian Refugees in Lebanon and Their Employment Profile, 2013. Beirut, ILO Regional Office for Arab States.

IMF (2018), Shadow Economies Around the World: What Did We Learn Over the Last 20 Years? IMF Working Paper/18/17. Washington, DC, International Monetary Fund.

KirKayak Kültür (ed.) (o. J.), The Dom: The "Other" Asylum Seekers from Syria. Şahinbey, Kırkayak Kültür.

Law I. (2014), Mediterranean Racisms: Connections and Complexities in the Racialization of the Mediterranean Region. Basingstoke, Palgrave Macmillan.

Malmö University \& UNICEF (ed.) (2017), Cross-sectoral Formative Research: Knowledge, Attitudes and Practice Study. Beirut, UNICEF Lebanon.

NAgEl T. (1986), The View From Nowhere. Oxford, Oxford University Press.

ODI (2018), The Jordan Compact: Lessons learnt and implications for future refugee compacts. London, Overseas Development Institute.

Pelek D. (2018), Syrian Refugees as Seasonal Migrant Workers: Re-Construction of Unequal Power Relations in Turkish Agriculture. Journal of Refugee Studies, [doi:10.1093/jrs/ fey050].

Rosch E. (1973), Natural Categories. Cognitive Psychology 4, pp. 328-350.

Rapport N. \& Stade R. (2014), Debating Irony and the Ironic as a Social Phenomenon and a Human Capacity. Social Anthropology 22 (4), pp. 443-478.

UNHCR (ed.) (2017), Jordan: Operational Update, January 2017. Erreichbar unter $<$ https:// reliefweb.int/sites/reliefweb.int/files/resources/UNHCR\%20Jordan\%20Operational\%20 Update\%20January\%202017\%20FINAL.pdf>

UNHCR (ed.) (2018a), A Longing to Go Home, in Safety and Dignity: Intentions and Perceptions of Syrian Refugees in Lebanon About Their Future. Beirut, UNHCR Lebanon.

UNHCR (ed.) (2018b), Fourth Regional Survey on Syrian Refugees' Perceptions and Intentions on Return to Syria (RPIS). Amman, UNHCR MENA Bureau.

VASYR (2017), Vulnerabity Assessment of Syrian Refugees in Lebanon. Beirut, UNHCR, UNICEF, WFP. 
WFP (ed.) (2018), Refugees in Turkey: Comprehensive Vulnerability Monitoring Exercise (Round 2). Ankara, World Food Program.

Yassin N. (2018), 101 Facts and Figures on the Syrian Refugee Crisis. Beirut, Issam Fares Institute for Public Policy and International Affairs, American University of Beirut.

Yildiz Y. (2015), Nowhere to Turn: The Situation of Dom Refugees from Syria in Turkey. Budapest, European Roma Rights Centre. 